\title{
IPv6 Performance and Reliability*
}

\author{
Warren Matthews \\ Stanford Linear Accelerator Center, Stanford University, Stanford, California 94309
}

\author{
Invited talk presented at IPv6-2000, Washington, Wash. D.C., USA \\ 10/19/2000-10/20/2000
}

\footnotetext{
Work supported by Department of Energy contract DE-AC03-76SF00515.
} 


\section{IPv6 Performance and Reliability}

Warren Matthews, SLAC.

Presented at the IPv6-2000 Conference, Washington DC, October 20, 2000. 


\section{Networking for HENP}

- BaBar Collaboration at SLAC

- International Collaboration

- 10 Countries

- Currently data is written to the database at $32 \mathrm{MBps}$

- Petabytes of data during the lifetime of the experiment

- Advanced Network users requiring Applications

- Experience tells us the rest of the Internet is only a few years behind.

- The LHC will be an order of magnitude bigger. 


\section{A Place for IPv6?}

- IPv6 not a solution, but possibly may provide advantages for Physics

- Developing Countries (address challenged).

- QoS

- IPSec/Security Features

- Porting of Physics Tools 


\section{IPv6 Deployment at SLAC}

- Native IPv6 Connection from ESnet

- Small amount of bandwidth carved off

- 2001:400:0808::/48

- SLAC Switched Network

- Cisco, Linux, FreeBSD, Solaris. 


\section{PingER}

- Started in 1995 at SLAC

- DoE/MICS, ESnet, ICFA-SCIC, XIWT, IAEA

- Simple ping end-to-end Monitoring

- 32 Monitoring Sites

- 420 Remote Sites in 72 Countries

- See May issue of IEEE Communications magazine. 


\section{PingERv6}

- Red Hat Linux with inet-apps from inner.net

- Data Collected from SLAC

- November and December 1999

- April to September 2000 (6 Months)

- Data Collected from 6TAP

- mid-June to mid-September 2000 (3 Months) 


\section{PingERv6 Sites}

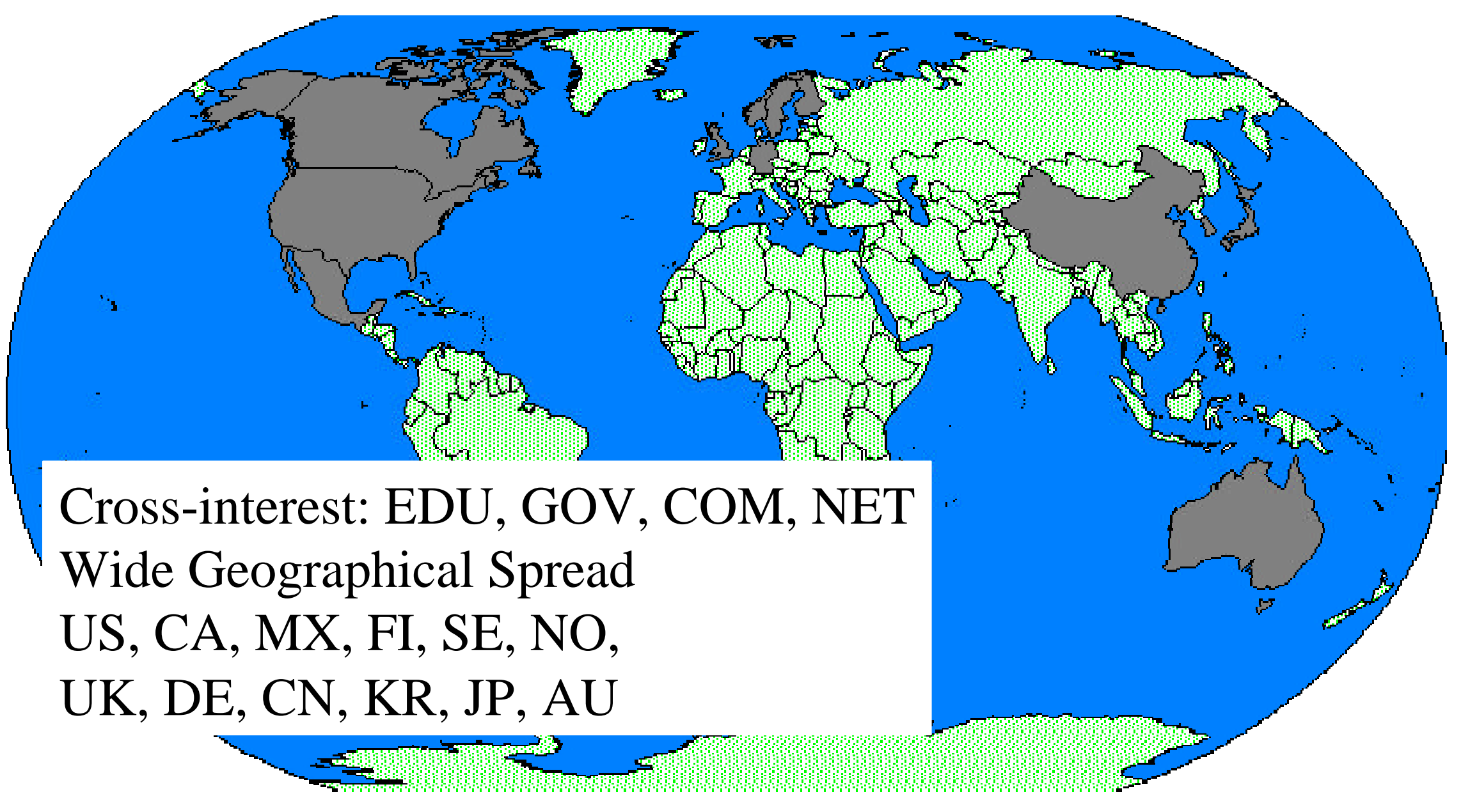




\section{Performance Summary}

- Unreachable decreasing

- Usually requires someone to notice.

- Typically at least 50\% are Good or Acceptable

- Indicates high traffic load on many links

- Better performance in the summer

Really measuring the performance of the Tunnel! 


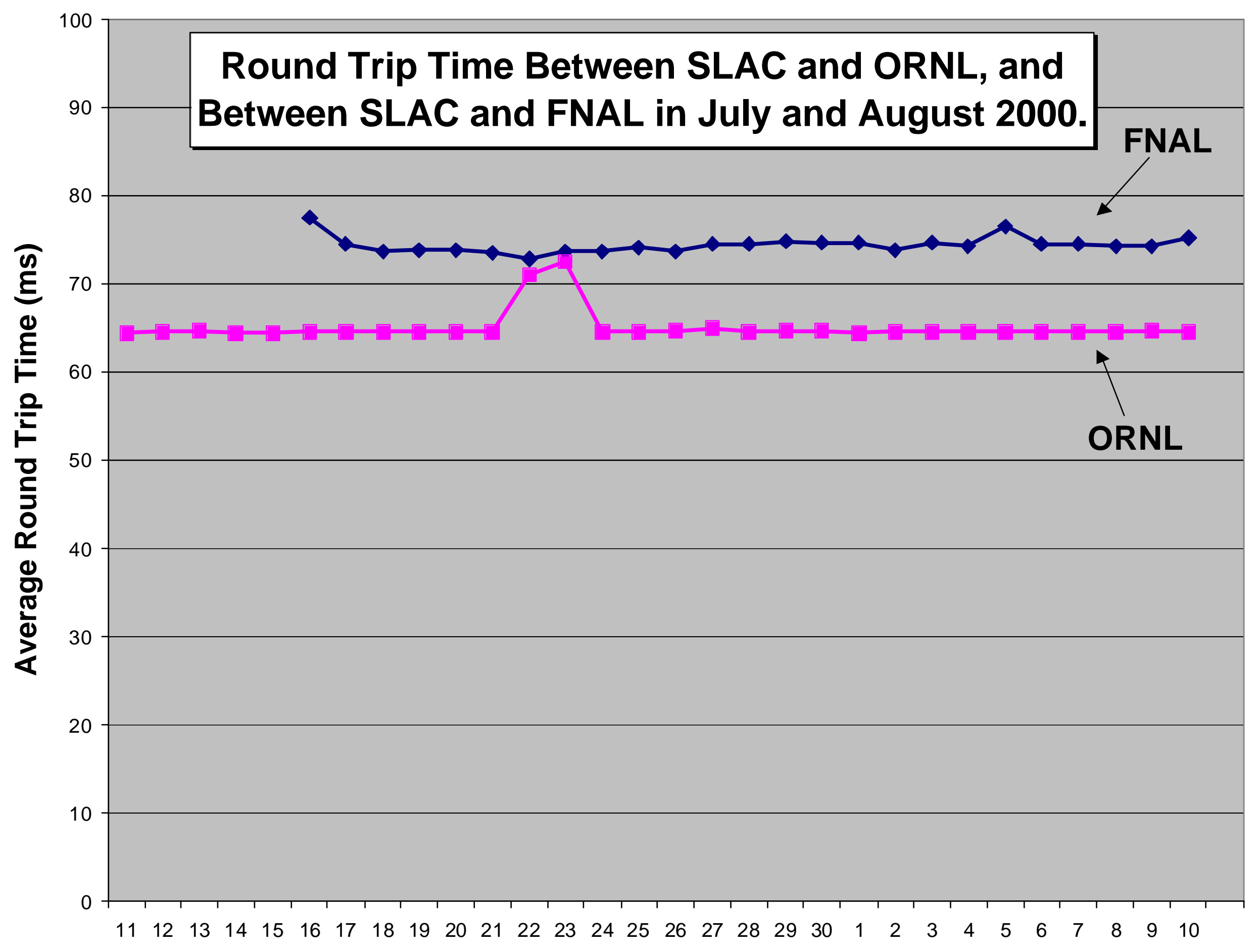




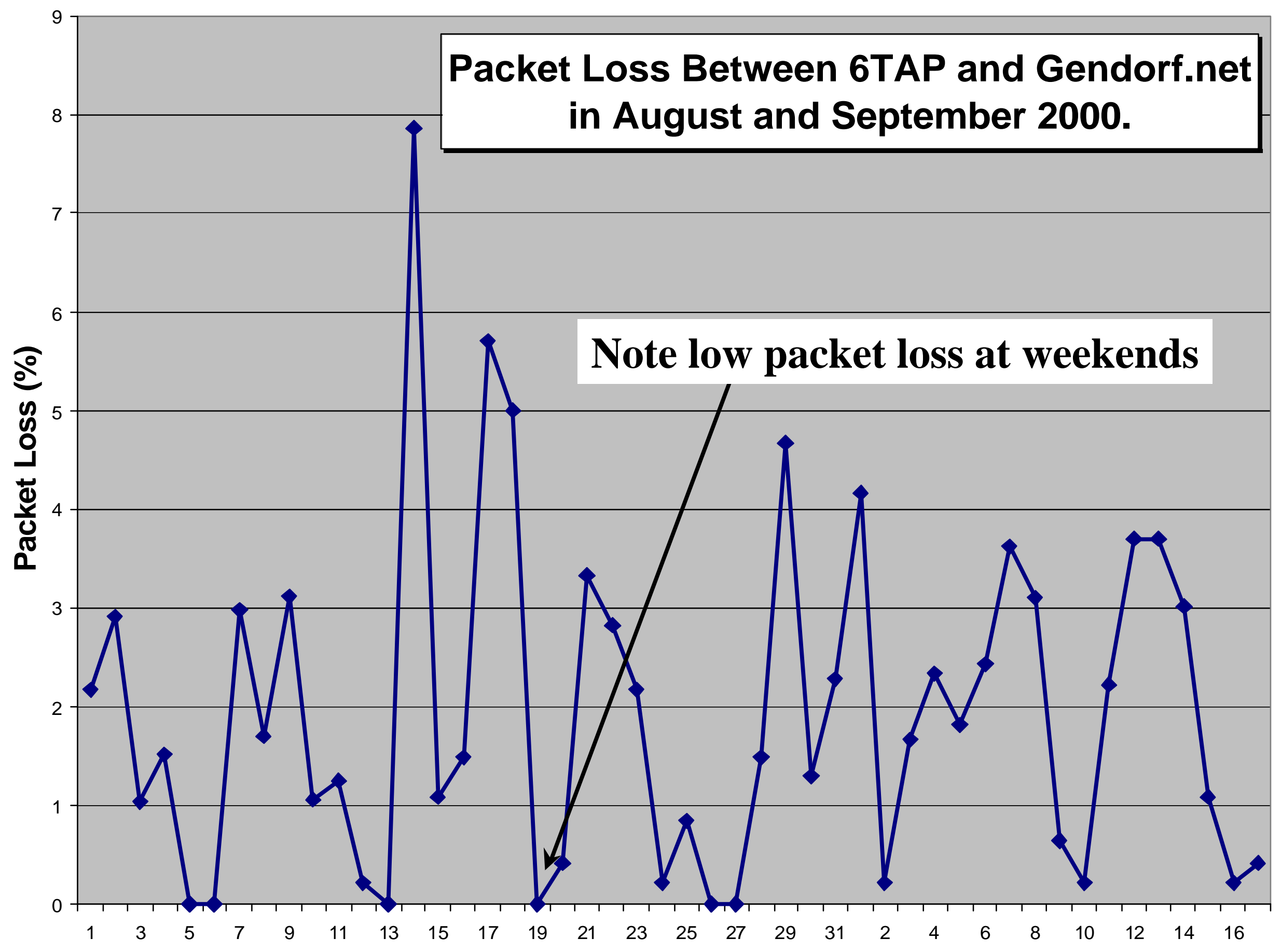




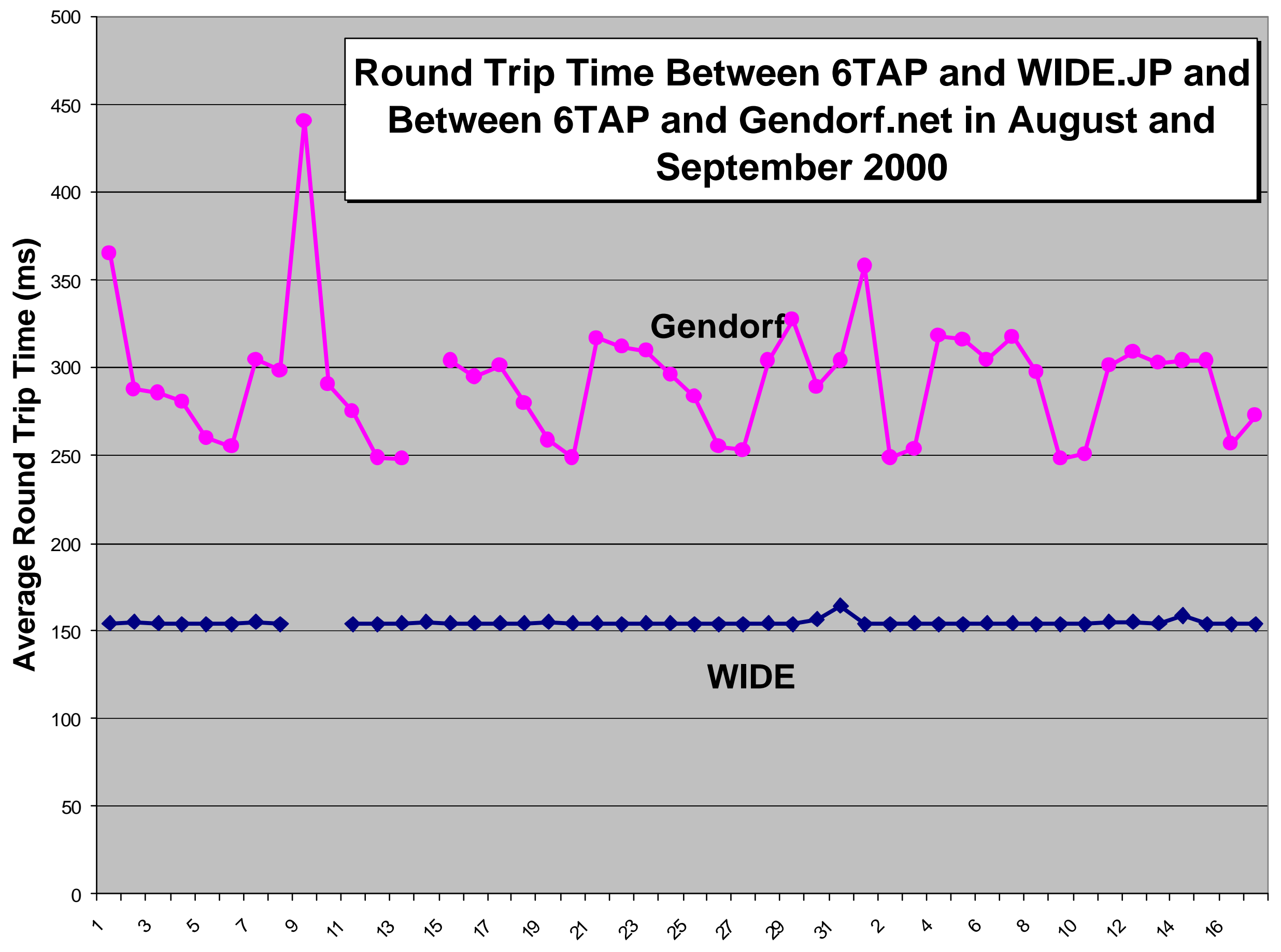




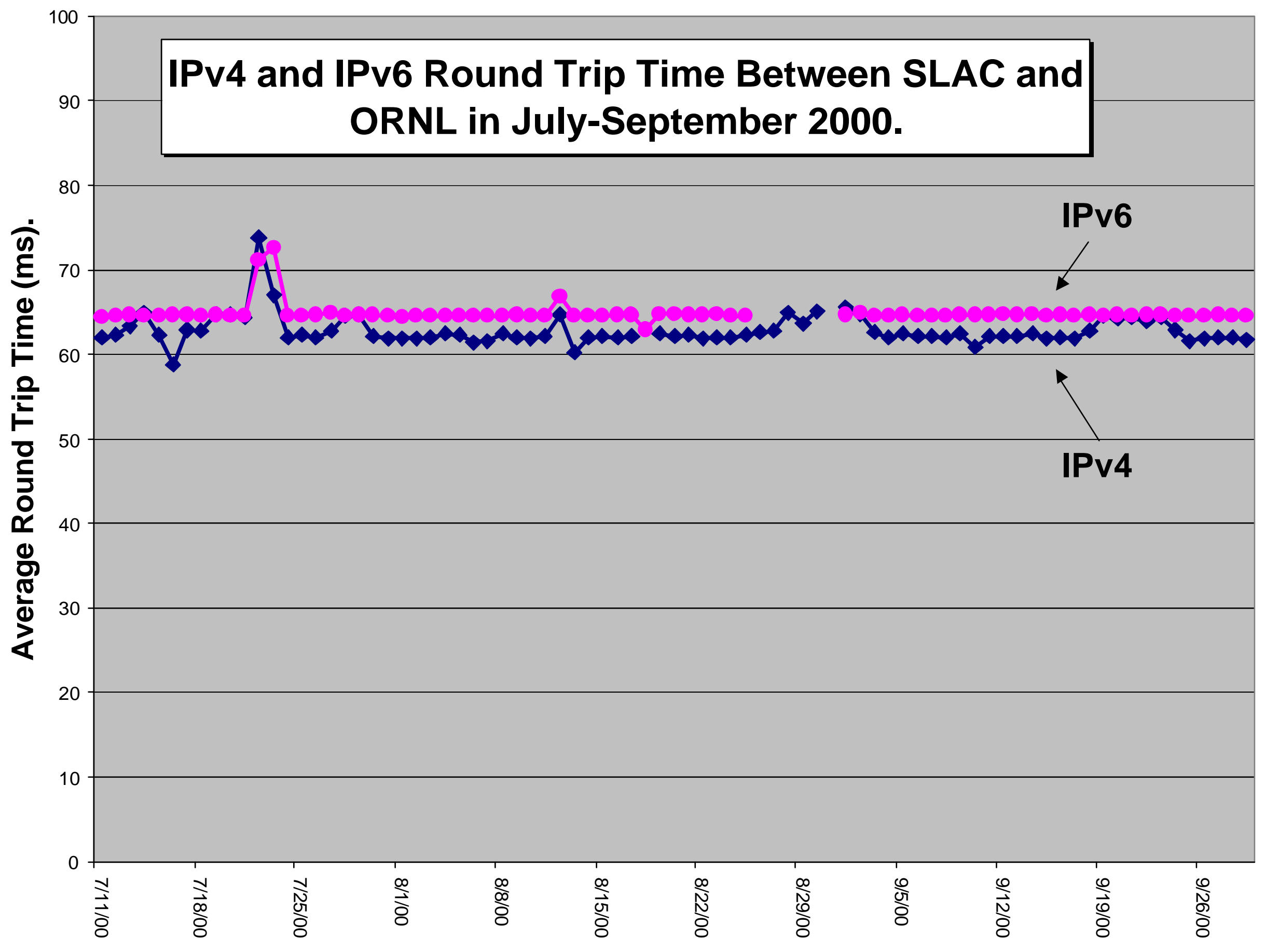




\section{Conclusions}

\section{On Performance and Reliability}

- Not surprisingly, many of the same performance issues as v4

- Expect IPv6 to do better than v4 in at least some situations

- Tunnels can work well

- Bottlenecks are everywhere 


\section{Conclusions For HENP.}

- Can't yet say that IPv6 features are going to dramatically improve the lives of physicists.

- If you build it, they will come.

- IPv6 is all over the world, just like the High Energy and Nuclear Physics Community. 


\section{Further Work}

- Further Deployment of machines and applications

- Porting, especially physics applications.

- Experiment, especially with collaborators in developing countries

- File Transfers 


\section{Thanks !}

- To all who volunteered

- Bob Fink, Becca Nitzan and ESnet

- IPv6 team at Viagenie

- The 6Bone mailing list 


\section{Further Information}

- http://www-iepm.slac.stanford.edu (IPv4)

- http://vvvvvv.slac.stanford.edu (IPv6)

- iepm@slac.stanford.edu (IPv4 only :)).

- SLAC Tech-Pub 8642 


\section{Any Questions ?}

